\title{
REDES NEURAIS ARTIFICIAIS: UTILIZAÇÃO DO ALGORITMO RETROPROPAGAÇÃO PARA CLASSIFICAÇÃO DE GRUPOS EM BIOSSISTEMAS, PARTE 1: INTRODUÇÃO TEÓRICA.
}

\author{
Beatriz Santos Bisi ${ }^{1}$ \\ Alfredo Bonini Neto ${ }^{2}$ \\ Carolina dos Santos Batista Bonini ${ }^{3}$
}

\section{RESUMO}

Redes neurais artificiais são modelos computacionais que consistem na semelhança da maneira como um organismo vivo manipula as informações recebidas e com isso, possuem capacidade de aprendizado, adaptabilidade e generalização do conhecimento. Além disso, o modelo de processamento de uma Rede Neural Artificial é baseado no paralelismo, assemelhando-se como o cérebro lida com as informações recebidas por seus neurônios. O presente trabalho foi dividido em duas partes, em que nesta primeira descreve o estudo da rede neural artificial Retropropagação (backpropagation) como um classificador de grupos, utilizando dois processos básicos desempenhados por uma rede neural artificial, as fases de treinamento ou aprendizado e a fase de operação. O objetivo principal é mostrar o processo de funcionamento dessas fases. Na parte II, é apresentado aplicações da metodologia para classificar grupos, tipos de frutas e solos.

PALAVRAS-CHAVE:Neurônio artificial, Classificação de grupos, Retropropagação, Treinamento.

\section{ARTIFICIAL NEURAL NETWORKS: ALGORITHM BACKPROPAGATION USE FOR CLASSIFICATION OF GROUPS IN BIOSYSTEMS, PART 1: INTRODUCTION THEORY.}

\begin{abstract}
Artificial neural networks are computational models that consist of similarity with a organism and thus, have learning ability, adaptability and generalization of knowledge. In addition, the processing model of an Artificial Neural Network is based on parallelism, resembling how the brain handles the information received by your neurons. This study was divided into two parts, where this first describes the study of artificial neural network Backpropagation as a classifier of groups using two basic processes performed by an artificial neural network, the phases of training or learning and operation phase. The main objective is to show the functioning process of these phases. In Part II, methodology of applications is presented to classify groups, types of fruits and soils.
\end{abstract}

KEYWORDS:artificial neuron, classificationgroups,Backpropagation, training

\footnotetext{
${ }^{1}$ Graduanda do curso de Engenharia de Biossistemas, Unesp- Campus de Tupã. beatriz_bisi@hotmail.com

${ }^{2}$ Professor Assistente Doutor do curso de Engenharia de Biossistemas, Unesp- Campus de Tupã. bonini@tupa.unesp.br

${ }^{3}$ Professora Assistente Doutora do curso de Engenharia Agronômica, Unesp- Campus de Dracena. carolbonini@dracena.unesp.br
} 


\section{REDES NEURONALES ARTIFICIALES: USO BACKPROPAGATION ALGORITMO PARA LA CLASIFICACIÓN DE LOS GRUPOS EN BIOSYSTEMS, PARTE 1: INTRODUCCIÓN TEORÍA.}

\section{RESUMEN}

Redes neuronalesartificialesson modelos computacionales que consistenenlasimilitud de la forma de un organismo vivo que maneja lainformaciónrecibido y generalizacióndelconocimiento, laadaptabilidad y capacidad de aprendizaje. Además, el modelo de procesamiento de una red neuronal artificial sebasaen paralelismo, parecido como elcerebro se ocupa de lainformaciónrecibida por lasneuronas. Este trabajo se dividióendos partes, que en esta primeradescribeelestudio de lared neuronal artificial Backpropagation como unclasificador de los grupos, utilizando dos procesos básicos efectuados por una red neuronal artificial, las fases de entrenamiento o fase de aprendizaje y operación. El objetivo principal es mostrar elproceso de funcionamiento de estas fases. Enla parte dos, sonaplicaciones de lametodología para laclasificación de los grupos, tipos de frutas y suelos.

PALABRAS CLAVE: neurona artificial, grupos de clasificación, Backpropagation, capacitación.

\section{INTRODUÇÃO}

As redes neurais artificiais (RNA) são sistemas computacionais adaptativos inspirados nas características de processamento de informação semelhante ao neurônio biológico de organismos inteligentes. Constituído de unidades de processamento simples, que têm a propensão natural para armazenar conhecimento experimental e torná-lo disponível para o uso. Essas redes permitem produzir saídas adequadas para entradas que não estavam presentes durante o treinamento.

Esses sistemas se assemelham ao cérebro humano de forma que o conhecimento de ambos são adquiridos a partir de seu ambiente através de um processo de aprendizagem e pela força de conexão entre os neurônios, no cérebro, e as redes, nos sistemas, denominados pesos sinápticos, os quais são utilizados para armazenar o conhecimento adquirido.

Nos últimos anos, publicações de trabalhos envolvendo redes neurais artificiais (RNA) têm crescido gradativamente no Brasil. SOARES et al (2014), objetivaram utilização de redes neurais artificiais para predição da produtividade da cultura do feijão, BUCENE \& RODRIGUES (2004), utilizaram as redes neurais artificiais para avaliação de produtividade do solo, visando classificação de terras para irrigação e CURI et al(2014), utilizaram redes neurais artificiais na predição da vazão ideal de exaustores em aviários de frango de corte com diferentes tipologias. 
E no exterior tem-se publicações de BEUCHERA et al (2015), que utilizaram as redes neurais artificiais para mapeamento de solo e caracterização das propriedades do solo relevantes para o planejamento ambiental; ANAGU et al (2009), utilizaram as redes neurais artificiais para desenvolver modelos de sorção (conjunto dos fenômenos de absorção, adsorção e dessorção) em função de propriedades básicas do solo e SILVEIRA et al (2013), manipularam as redes neurais artificiais do tipo multicamadas com o objetivo de prever solos de acordo com seus atributos.

Esses estudos devem-se ao grande poder de aplicações destas metodologias em diversas áreas. As redes neurais artificiais são capazes de extrair informações não apresentadas de forma explícitas através de exemplos; são adequadas para a análise de sistemas com incertezas, sendo usadas como alternativas aos métodos estatísticos.

Com isso, nesta primeira parte do trabalho é apresentado o processo de funcionamento da rede neural artificial, o algoritmo Retropropagação (backpropagation), o qual consiste em multicamadas não-recorrentes, com treinamento supervisionado.

\section{OBJETIVO}

O objetivo desta primeira parte é estudar o processo de funcionamento da rede neural artificial Retropropagação (backpropagation), fase de treinamento para obtenção dos pesos e minimização de erros, e fase de operação para classificação de grupos.

Fazer também um levantamento bibliográfico do uso de redes neurais em biossistemas (classificação de grupos e aplicação em solos)

\section{METODOLOGIA}

Baseado na relação das redes neurais artificiais com os neurônios biológicos, em que nos neurônios biológicos há os dendritos que recebem as informações, o corpo celular que soma e assimila essa informação e o axônio que 
transmite tal informação resultada pelos impulsos nervosos, assim ocorrendo à conexão entre os neurônios pela sinapse (representados na figura 1).

Figura1: Representação de um neurônio biológico.

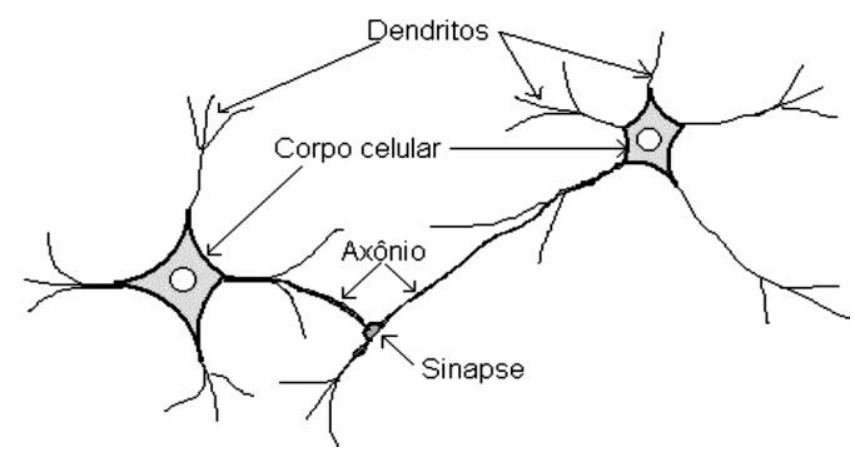

Fonte: Minussi (2003)

Portanto, em meio a essa comparação, as redes neurais artificiais devem conter entradas, que seriam as informações, os pesos que seriam determinantes da sinapse (MCCULLOCH e PITTS, 1943), a assimilação dessa informação e uma saída obtida de tal processo, figura 2.

Figura 2: Modelo artificial de neurônio biológico.

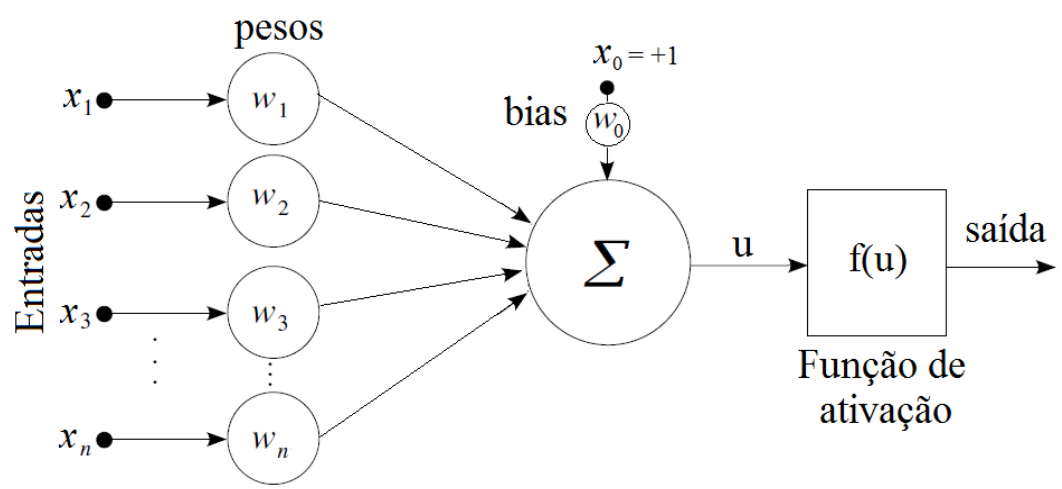

Fonte:Minussi (2003) 
Onde o $\sum$ representa o somatório dos produtos das entradas pelos respectivos pesos, ou seja, $u=\sum_{i=1}^{n} \mathbf{x}_{i} \mathbf{W}_{i}+$ bias e $\mathrm{f}(\mathrm{u})$ é a função de ativação para obter a saída, existem várias funções de ativação como mostrado na figura 3:

Fugura3: Tipos de funções de ativação.

\begin{tabular}{|c|c|c|c|}
\hline Função relé (degrau) & Função lógica & Função sigmoide (1) & Função şigmoide (2) \\
\hline & & \\
\hline &
\end{tabular}

$\lambda=$ inclinação, $\lambda$ e $t$ constantes reais

Fonte: Autores

As RNA são classificadas da seguinte forma: Redes recorrentes e redes não recorrentes (feedforward). As redes recorrentes são aquelas que contêm laços de realimentação, ou seja, contém conexões das saídas de uma determinada camada para a entrada da mesma ou de camadas anteriores e redes não recorrentes, são aquelas que não possuem laços de realimentação, caracteriza-se estruturalmente por estar disposta em camadas, figura 4 e 5. 
Figura 4: RNA recorrente

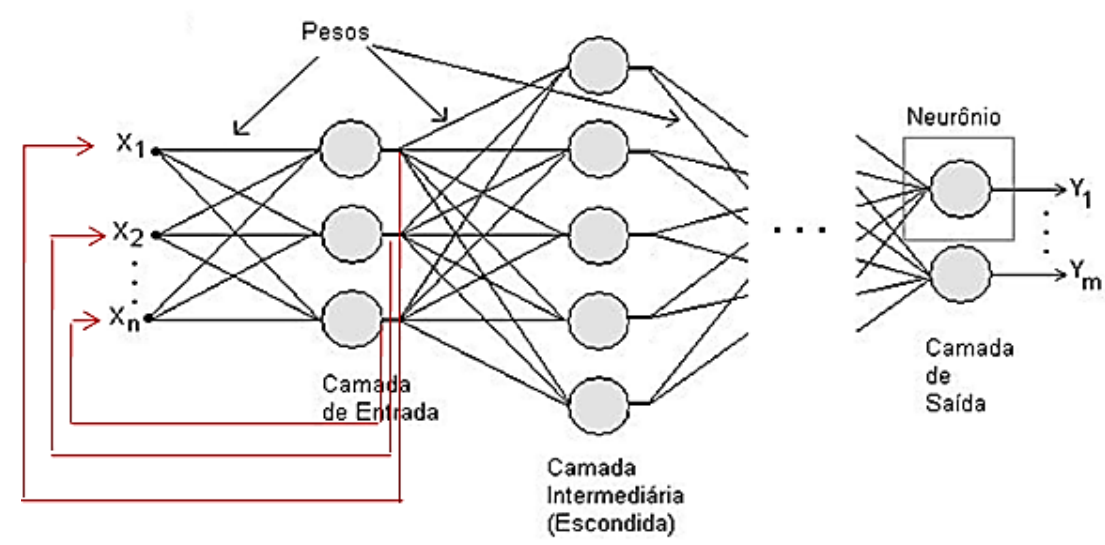

Fonte: Minussi (2003)

Figura 5: RNA não recorrente

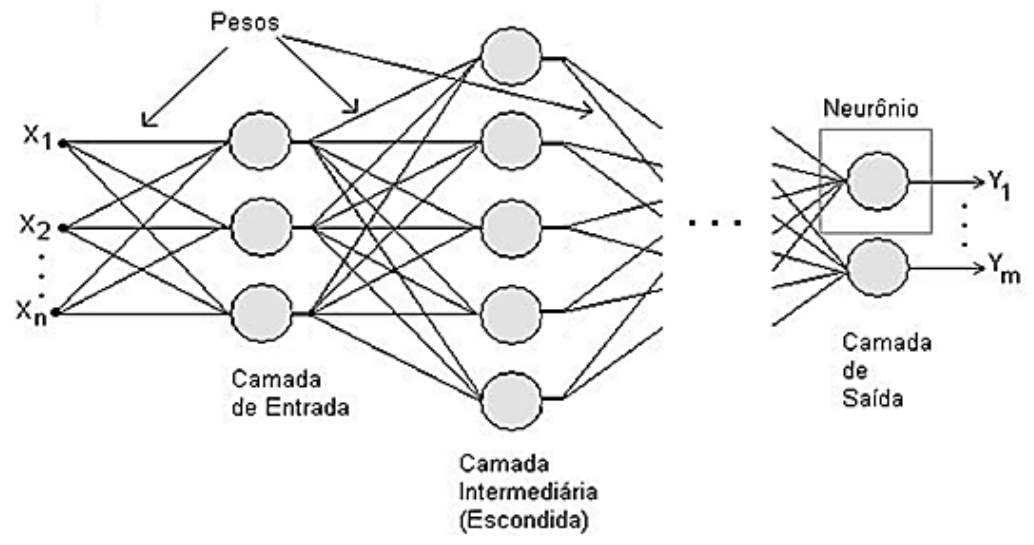

Fonte: Minussi (2003)

\section{ALGORITMO RETROPROPAGAÇÃO (BACKPROPAGATION)}

Relatada em 1986 por Rumelhart. Hinton e Williams, o algoritmo de Retropropagaçãoteve seu funcionamento explicado, o qual se fez o algoritmo de aprendizagem mais popular para treinamentos de redes neurais artificiais algoritmo perceptron de multicamadas. Após o relato em 1986 foram descobertos anotações sobre o assunto com datas desde 1969, porém, Rumelhart, Hinton e Williams levaram créditos pelo algoritmo de retropropagação por proporem a sua utilização para a aprendizagem de máquina e por demonstrarem como isto poderia funcionar.

O algoritmo retropropagaçãoé um algoritmo de multicamada, pois consiste de vários neurônios em mais de uma camada, com pesos sinápticos ajustáveis e bias; como mostrado na figura 6. Possui treinamento supervisionado, com erro 
calculado e minimizado para obtenção dos pesos sinápticos de todas as camadas. Baseia-se contendo entradas e saídas específicas para cada entrada.

Figura 6. Representação da rede Retropropagação

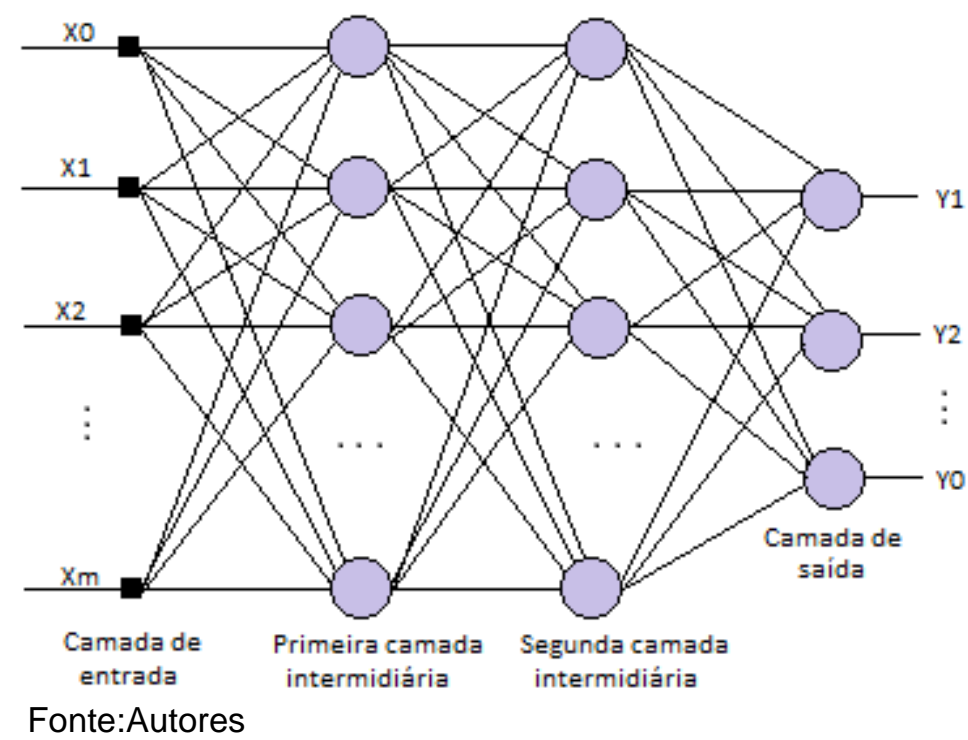

Simula um neurônio com entradas e pesos que, ajustados teriam a capacidade de aprender a se comportar de determinada forma. Porém, para que as saídas sejam as desejadas é necessário que haja a fase de treinamento para ajustamento dos pesos para a fase de operação ser adequada.

\section{FASE DE TREINAMENTO}

A propriedade mais importante das redes neurais é a habilidade de aprender e com isso melhorar seu desempenho. Isso é feito através de um processo iterativo de ajustes aplicados a seus pesos $\mathbf{W}$ que correspondem ao treinamento. Existem dois tipos de treinamento, supervisionado e o não supervisionado.

Treinamento Supervisionado consiste no ajuste de pesos de uma rede neural para fornecer saídas desejadas. O treinamento supervisionado necessita de um de vetor de entrada e vetor alvo representando a saída desejada, figura 7. 
Figura 7: Treinamento supervisionado

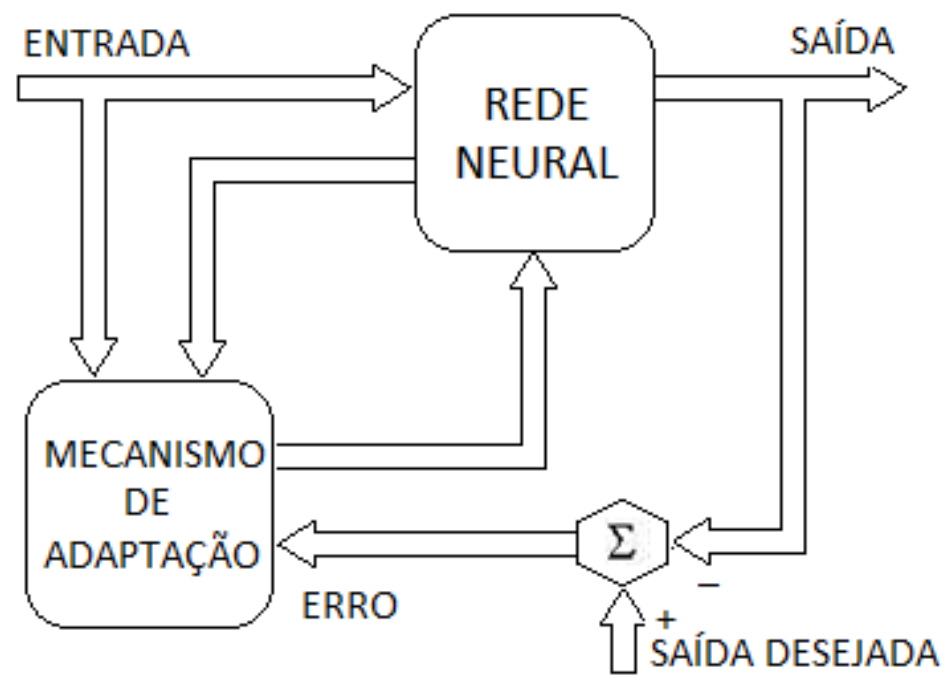

Fonte: Autores

Treinamento não supervisionado consiste no ajuste de pesos de uma rede neural, levando-se em conta somente 0 conjunto de padrões de entrada (treinamento auto-organizável), conforme a figura 8.

Figura 8: Treinamento não supervisionado

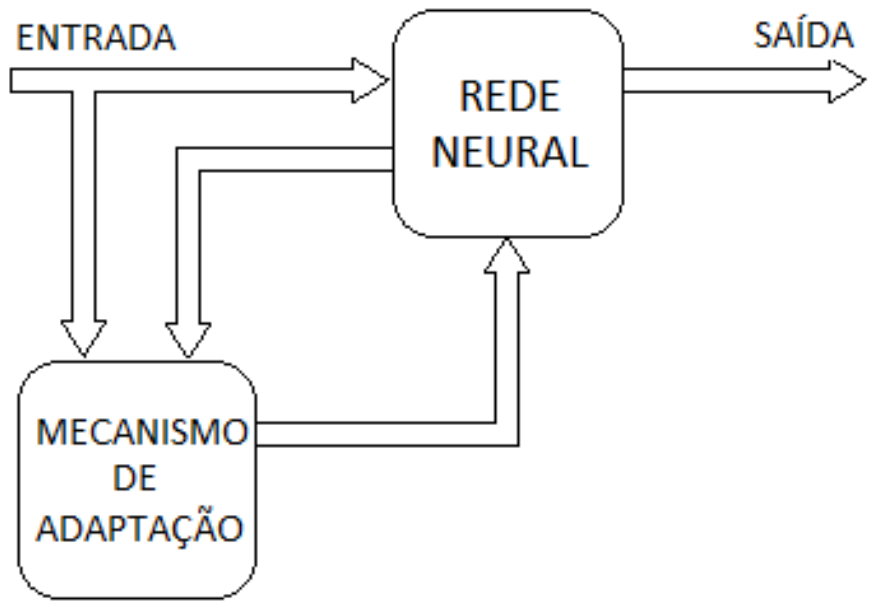

Fonte: Autores

A fase de treinamento do algoritmo Retropropagaçãoconsiste na apresentação de entradas e saídas desejadas para essas entradas. O erro é calculado para cada saída (diferença entre a saída desejada e a saída obtida), depois se calcula o erro propagado no sentido contrário, através da rede associada à derivada parcial do erro quadrático de cada elemento com relação aos pesos e, finalmente, ajustando os pesos de cada elemento. Assim, um novo padrão é 
apresentado, em que o processo continua até o erro ser menor do que o préestabelecido. Esse processo define os ajustes dos pesos sinápticos, minimizando o erro quadrático da rede.

\section{FASE DE OPERAÇÃO}

Após serem definidos os pesos sinápticos da rede Retropropagação, através do processo de treinamento, a rede estará apta a classificar padrões de acordo com a sua aplicação anteriormente estabelecida. Portanto, na fase de operação ou execução, o Retropropagação produz para cada amostra, as respectivas saídas desejadas, e, além disso, se torna capaz de classificar amostras que não fizeram parte da fase de treinamento, isto é, a rede possui a habilidade de generalizar o conhecimento adquirido.

\section{CONCLUSÃO}

As redes neurais artificiais são tipicamente usadas em reconhecimento de padrões, onde informação das características da classe analisada é apresentada à rede e a tarefa é categorizar o padrão característico da entrada como pertencente a uma ou mais classes.

Nesta primeira parte do trabalho foi apresentado a parte teórica do algoritmo Retropropagação (backpropagation) fase de treinamento, explicando os ajustes dos pesos para a classificação de grupos; e a fase de operação, que consiste na habilidade de classificar grupos. Também foi feito uma revisão bibliográfica da sua aplicação em biossistemas (classificador de frutas e solos).

\section{REFERÊNCIAS}

ANAGU, I.; INGWERSEN, J.; UTERMANN, J. and STRECK, T. Estimation of heavy metal sorption in German soils using artificialneural networks.Geoderma, v. 152, Issues 1-2,15, p. 104-112. 2009.

BEUCHERA, A.; SIEMSSENA, R.; FRÖJDÖA, S.; ÖSTERHOLMA, P.; MARTINKAUPPIB, A. and EDÉNB, P. Artificial neural network for mapping and characterization of acid sulfate soils: Application to Sirppujoki River catchment, southwestern Finland. Geoderma. v. 247-248, p. 38-50, 2015. 
BUCENE, L. C.; RODRIGUES, L. H. A. Utilização das Redes Neurais Artificiais para avaliação da produtividade do solo, visando classificação de terras para irrigação.Revista Brasileira de Engenharia Agrícola e Ambiental, Campina Grande, v. VIII, n. 2-3, p. 326-329, 2004.

CURI, T. M. R. de C.; DE MOURA D. J.; MASSARI, J. M.; VERCELLINO, R. A.; SABINO, L. A. Redes Neurais na predição da vazão ideal de exaustores em aviários de frango de corte com diferentes topologias. In:Congresso Brasileiro de Engenharia Agrícola, 43., 2014.

MCCULLOCH W. S. e Pitts, W. A. Logical Calculus of the Ideas Immanent in Nervous Activity, Bulletin of Mathematical Biophysics, 1943, No. 9, pp. 127-147.

MINUSSI C. R. Redes Neurais: Introdução e principais conceitos. Faculdade de Engenharia de llha Solteira. Notas de aula - UNESP. 2003.

SILVEIRA, C. T.; OKA-FIORI, C.; SANTOS, L. J. C.; SIRTOLI, A. E.; SILVA, C. R.; BOTELHO, M. F. Soilpredictionusing artificial neural networks andtopographicattributes. Geoderma, v. 195-196, p. 165172, 2013.

SOARES, F. C.; RUSSI, J. L.; ROBAINA, A. D. PEITER, M. X.; PARIZI, A. R. C.Uso de Rede Neural Artificial para predição da produção na cultura do feijoeiro. In: Congresso Brasileiro de Engenharia Agrícola, 43, 2014. 